\title{
Clot retrieval and acute stroke care
}

\author{
Resource distribution in stroke care must be rational and evidence-based, \\ not driven by media coverage
}

$\mathrm{T}$ here has been a sudden upsurge of interest in the availability of endovascular clot retrieval (ECR) for stroke treatment (Box). Recent newspaper articles published in New South Wales ${ }^{1,2}$ highlight the potential benefits as well as the financial and logistical challenges of providing around the clock ECR services in a vast country like Australia.

Many well designed randomised trials have demonstrated the efficacy and safety of $\mathrm{ECR}_{,}{ }^{3}$ but the evidence of benefit, although dramatic in some cases, is confined to patients whose ECR procedure begins within 6 hours of symptom onset, ${ }^{4}$ with or without intravenous thrombolysis. However, before 24/7 ECR services can operate in Australia, individual health services need to examine the challenges of transporting eligible stroke patients from the emergency departments wherever they may be - remote, regional or urban - to a comprehensive stroke centre within the required time window. This potentially means covering vast distances several hundreds of kilometres in some cases - in a matter of hours. A detailed analysis then needs to demonstrate that the benefits of a rapid ECR service justify funding over many other competing health care needs. Victoria, the third smallest Australian health jurisdiction (after the Australian Capital Territory and Tasmania) with the second highest population, ${ }^{5}$ has set an example by starting a statewide $24 / 7$ ECR service. However, it is unclear at this stage whether a similar service would be feasible in larger states with lower population densities, given the similar challenges for ECR service provision in the United States and Canada. ${ }^{6}$

Currently, there are no 24/7 statewide ECR pathways in New South Wales. In the Australian Capital Territory, the health service is establishing a 24/7 ECR service in 2017, although many hurdles still remain.

However, even within stroke care, there are other, perhaps more immediate, needs. Currently, there are large gaps in our ability to deliver basics of stroke care, particularly in regional Australia. For example, a 2015 national audit of acute stroke services coordinated by the Stroke Foundation showed that only $67 \%$ of patients were admitted into a stroke unit, ${ }^{7}$ even though patients admitted to stroke units with any type of stroke,

Yun Tae Hwang ${ }^{1,2}$

Yash Gawarikar ${ }^{2,3}$

IUCL Institute of Neurology, London, UK

2 Australian National University

Canberra, ACT

3 Calvary Public

Hospital Bruce,

Canberra, ACT ischaemic and haemorrhagic, are more likely to be alive,

living at home and independent 1 year after their stroke.

Another area that could be improved is availability of advanced imaging - currently, as a result of lack of expertise and local department policy, too many stroke patients, even in urban centres, do not benefit from multimodal imaging such as computed tomography (CT) angiogram or CT perfusion scans. These scans can demonstrate the presence and location of a clot within the cerebral vasculature, as well as the size of the penumbra (the area of the brain at risk of infarction without urgent revascularisation), to identify patients who may
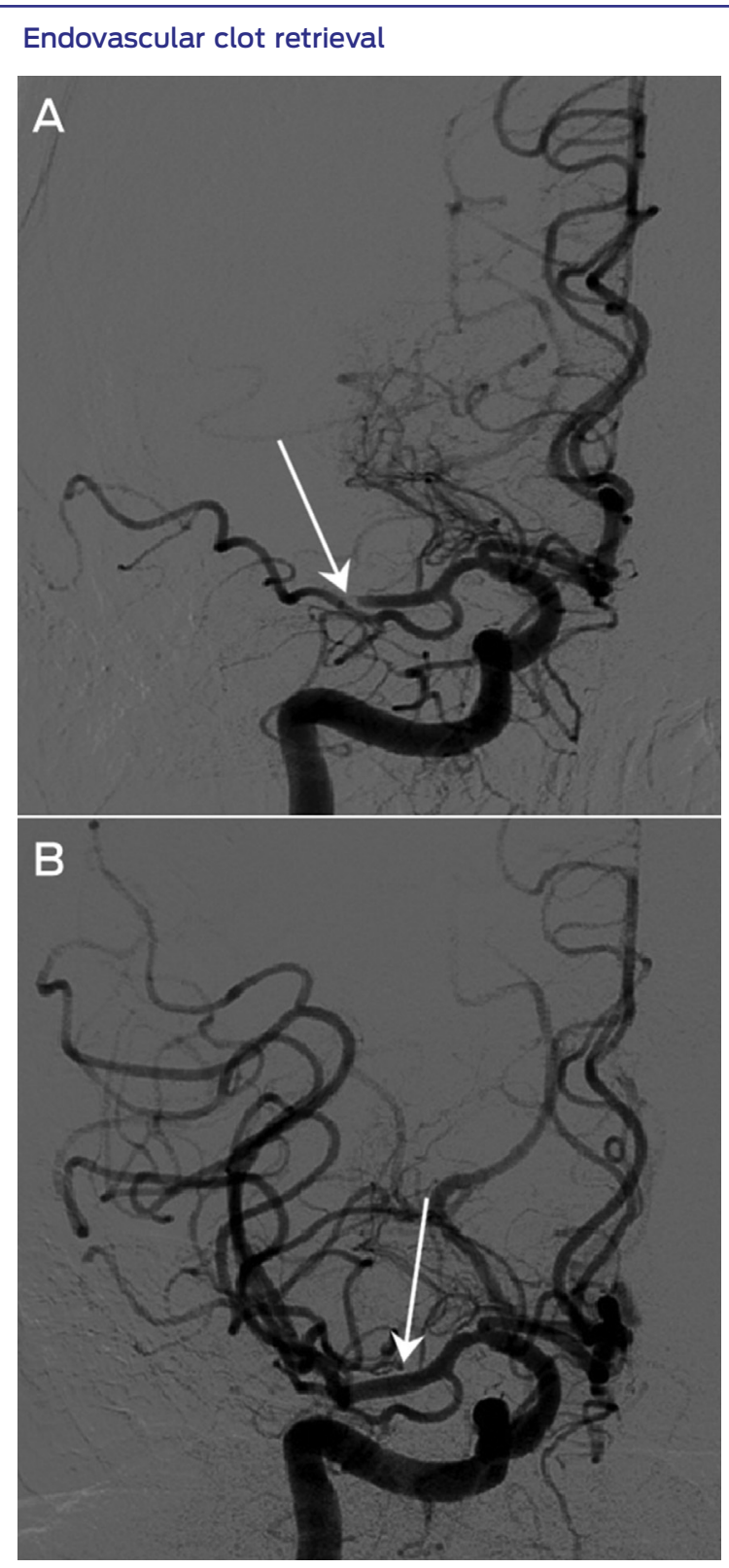

Cerebral angiograms showing (A) occlusion of the proximal right middle cerebral artery (arrow), and (B) recanalisation of the same artery after clot retrieval (arrow). Images courtesy of Dr Shivendra Lallo, Canberra Hospital, ACT.

benefit from aggressive and more invasive treatments such as ECR.

Further, intravenous thrombolysis - a proven ${ }^{10}$ and more easily accessible therapy than ECR - is currently not an option for many stroke patients who would be eligible. The national thrombolysis rate is currently languishing at $7 \%$, unchanged from $2011 .{ }^{7}$ Some work has already been done to improve this by establishing $24 / 7$ acute stroke teams and forming regional acute stroke 
networks where a large hospital provides thrombolysis expertise and support for the regional and rural hospitals. Setting up a statewide ECR service without widely available capacity to optimally assess, image, treat and transport stroke patients risks squandering precious resources without clear benefits for the majority who do not live in the immediate vicinity of an ECR centre.

Delivering a world class stroke service is complex; the constantly advancing evidence base means that the entire service is always playing catch up. It is important to foster a provision and funding model for rational, holistic and flexible stroke services that puts patient outcomes first and covers all aspect of stroke care - not just the acute reperfusion therapies of intravenous thrombolysis and ECR but also stroke unit care and specialist neurorehabilitation. There is much work to be done, given that only one in 87 stroke units qualifies as a comprehensive stroke service, ${ }^{7}$ only $40 \%$ of stroke units routinely utilise established guidelines, care plans and protocols, ${ }^{7}$ and one in three patients is discharged from hospital without any preventive medications. ${ }^{\text {? }}$ Focusing the discussion about stroke care only on the availability of ECR - a necessary but complex and costly intervention which will benefit only a small proportion of stroke patients - diverts resources from wider and more fundamental needs in stroke care and does not serve the best interests of our patients.

Competing interests: No relevant disclosures.

Provenance: Not commissioned; externally peer reviewed.

( 2017 AMPCo Pty Ltd. Produced with Elsevier B.V. All rights reserved.

References are available online at www.mja.com.au. 
1 Alexander H. Stroke patients missing out on life-saving treatment after hours. Sydney Morning Herald 2016; 10 Oct. http://www.smh.com.au/

national/health/stroke-patients-missing-out-on-lifesaving-treatment-afterhours-20161005-grwlgh.html (accessed Oct 2016).

2 Brennan R. Stroke cases after hours in NSW 'left to die', specialist doctors not available. Daily Telegraph 2016; 29 Sep. http://www.dailytelegraph.com. au/news/nsw/stroke-cases-after-hours-in-nsw-left-to-die-specialistdoctors-not-available/news-story/bf9e93fa62421c33e3535d22e2067f47 (accessed Oct 2016).

3 Saver JL, Goyal M, van der Lugt A, et al. Time to treatment with endovascular thrombectomy and outcomes from ischemic stroke: a metaanalysis. JAMA 2016; 316: 1279-1288.

4 Powers WJ, Derdeyn CP, Biller J, et al. 2015 American Heart Association/ American Stroke Association focused update of the 2013 guidelines for the early management of patients with acute ischemic stroke regarding endovascular treatment: a guideline for healthcare professionals from the American Heart Association/American Stroke Association. Stroke 2015; 46: 3020-3035.
5 Australian Bureau of Statistics. Australian Demographic Statistics, Jun 2016 (ABS Cat. No. 3101.0)Canberra: ABS, 2016. http://www.abs.gov.au/ ausstats/abs@.nsf/PrimaryMainFeatures/3101.0?OpenDocument (accessed Mar 2017).

6 Smith EE, Schwamm LH. Endovascular clot retrieval therapy implications for the organization of stroke systems of care in North America. Stroke 2015; 46: 1462-1467.

7 Stroke Foundation. National stroke audit: acute services report 2015. Melbourne: Stroke Foundation, 2015. https://informme.org.au/-/media/ A3039E82D9FF4BC7803B5C67B42DBBB0.ashx?la=en (accessed Dec 2016).

8 Stroke Unit Trialists' Collaboration. Organised inpatient (stroke unit) care for stroke. Cochrane Database Syst Rev 2013; 9: CD000197.

9 Morgan $C D$, Stephens M, Zuckerman S, et al. Physiologic imaging in acute stroke: patient selection. Interv Neuroradiol 2015; 21: 499-510.

10 Wardlaw JM, Murray V, Berge E, del Zoppo GJ. Thrombolysis for acute ischaemic stroke. Cochrane Database Syst Rev 2014; 7: CD000213. 